\title{
Comorbidities and multimorbidity in rheumatic diseases
}

\author{
Anna Filipowicz-Sosnowska \\ Consultant at National Institute of Geriatrics, Rheumatology and Rehabilitation, Warsaw, Poland
}

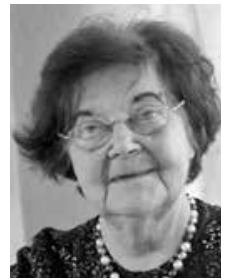

Recently, the significant negative impact of comorbidities on the course of rheumatoid arthritis (RA) and other inflammatory diseases of the joints has been underlined $[1,2]$.

A number of comorbidities may have individual impact on the course of rheumatic disease (RD) in individual patients and lead to the limitation of therapeutic options of both diseases. If the co-morbid disease is diagnosed early, properly treated, and monitored, its negative impact on the course of inflammatory RD, e.g. as RA, may be significantly reduced, which is important for the RD prognosis.

Considering the inflammatory pathophysiological process, the increased risk of diseases, such as: cardiovascular diseases (CVD), lungs and pleura diseases, osteoporosis, infection, depression, and cancers, the incidence of which in RA and other inflammatory RDs is significantly higher than in the general population, should be emphasised. However, the importance of other diseases, such as: diseases of the digestive tract, kidneys, diabetes, and liver diseases, should not be underestimated because they may lead to the deterioration of the patient's health and reduction of therapeutic options, which requires introduction of comprehensive care for such patients. The reasons for multi-organ involvement in patients with inflammatory joint diseases are not well understood.

It is assumed that the inflammatory process with a release of proinflammatory cytokines and chemokines plays an important role in that phenomenon. Other factors, such as: a reduction of physical activity, in some extreme cases even total immobilisation, obesity, muscle weakness, depression, and pharmacological therapy, mainly with glucocorticosteroids, are considered to be important reasons for the occurrence of diseases other than RD. There is a distinction between coexisting diseases related to RD (and inflammation) or its treatment and diseases independent of the underlying disease (coincidental). Patients with RA and comorbidities constitute the majority in the population of RA patients [3].
Comorbidities are also factors of "poor prognosis" in the course of RA [4]. They also correlate with worse response to treatment, and deterioration of functional capacity and quality of life $[4,5]$. Their occurrence increases mortality in RA patients [6]. It is clear that the problem of the coexistence of RD with other diseases is associated with higher socio-economic costs, i.e. more frequent hospitalisation, longer duration of hospitalisation, repeat visits to specialists, perioperative complications, and an increase indirect and direct costs of medical care [7]. The recent approach to RD known as "treat-to-target" (T2T) strategy, e.g. in treatment of RA or spondyloarthropathies, may be significantly hampered, and the assumed aims of therapies become impossible to achieve with the coexistence of other diseases [8].

Current recommendations for the RD management are based on the results of clinical trials, in which patients with comorbidities were generally not included.

The most frequently used assessment tools for comorbidities in RD are: the Charlson Comorbidities Index (CCI) [9], the Rheumatic Disease Comorbidity Index (RDCl) [10], and the Multimorbidity (MM) Index [11]. However, these methods are not adequate for the function, quality of life and risk of mortality evaluation. In a multimorbidity situation, it is difficult to assess the quality of life, a multidimensional health assessment questionnaire may be used in such instances [12].

The comorbidity and multimorbidity are often not distinguished as separate phenomena and are used interchangeably [13]. In the concept of comorbidity, RD, like $R A$, is central and coexistent diseases are related, being in some cases a consequence of the onset of RD. In the case of multimorbidity, the patient is at the centre and all diseases coexist and affect each other's course and the patient's condition. The assessment of comorbidities is based on the disease index and it determines the coexistence of any other disease. Multimorbidity covers all aspects of the patient's condition, including the interaction with the underlying disease and possible pathophysiological connections. Therefore, it is a broader con- 
cept than the concept of an accompanying/co-existing disease(s). In multimorbidity, the disease index is not defined, and all co-morbidities are equally important. The statistical data reveal a vital fact, i.e. the average frequency of patients with multimorbidity in the population is estimated to be around $25 \%$, whereas in patients with chronic arthritis the group reaches up to 75\% [13]. The discussion concerning the issue of which diseases should be included in the multimorbidity spectrum is still open. Currently, the list of coexisting diseases includes 40 items with high heterogeneity, without any selectivity criteria. The influence of multimorbidity on the patient's condition depends, which is understandable, on the number of chronic morbidities.

There are differences in the therapeutic strategy, depending on which theory: comorbidity or multimorbidity, is to be followed. In the case of comorbidities, the treatment is directed to the underlying disease, e.g. RA. The evaluation of the results of the RD treatment is based on the monitoring of disease activity using validated indicators such as DAS28 or SDAl and the disease index.

Clinical studies showed that the most common comorbidities in patients with chronic joint diseases include: hypertension (53\%), hypercholesterolaemia (47\%), CVD (24\%), obesity (36\%), and diabetes (16\%). The coexistence of these diseases often complicates or limits the recommended management of joint diseases, due to the interaction of drugs, polypharmacy, or drug intolerance. There is growing interest in a holistic approach to the treatment of patients with RDs. The recommendations of the EULAR 2016 initiative group [14] concern the development of the standardised methods for the assessment and reporting of comorbidities in everyday rheumatological practice. As a result of this consensus, the necessity to assess the frequency of occurrence of comorbidities in the course of RD, to screen for the most common diseases co-existing with inflammatory joint diseases, and to determine the risk of their occurrence and prevent co-morbid diseases, was established.

The main principles of conduct were formulated in the following three points:

1. Co-morbidities (cardiovascular system and gastrointestinal tract diseases, osteoporosis, depression, or cancer) should be carefully evaluated and treated in patients with RDs.

2. Rheumatologists, health professionals, and patients play a significant role in detecting and monitoring co-morbidities. It is recommended that an individual questionnaire be completed by patients, developed by the EULAR Group.

3. Co-morbidities should be covered by a specially developed program, which should be systematically updated every five years.
The author declares no conflict of interest.

\section{References}

1. Rupp I, Boshuizen HC, Roorda LD, et al. Poor and good health outcomes in rheumatoid arthritis: the role of comorbidity. J Rheumatol 2006; 33: 1488-1495.

2. Michaud K, Wolfe F. Co-morbidities in rheumatoid arthritis. Best Pract Res Clin Rheumatol 2007; 21: 885-906.

3. Aletaha D, Dörner T. Considering comorbidity in managing rheumatic diseases: going where trials cannot go. Arthritis Res Ther 2011; 13: 116.

4. Smolen JS, Landewé R, Bijlsma J, et al. EULAR recommendations for the management of rheumatoid arthritis with synthetic and biological disease-modifying antirheumatic drugs: 2016 update. Ann Rheum Dis 2017; 76: 960-977.

5. Radner H, Smolen JS, Aletaha D. Impact of comorbidity on physical function in patients with rheumatoid arthritis. Ann Rheum Dis 2009; 69: 536-541.

6. Menotti A, Mulder J, Nissinen A, et al. Prevalence of morbidity and multimorbidity in elderly male populations and their impact on 10-year all-cause mortality: The FINE study. J Clin Epidemiol 2001; 54: 680-686.

7. Wolff JL, Starfield B, Anderson G. Prevalence, expenditures, and complications of multiple chronic conditions in the elderly. Arch Intern Med 2002; 162: 2269-2276.

8. Nikiphorou E, Ramiro S, van der Heijde D, et al. Association of comorbidities in spondyloarthritis with poor function, work disability, and quality of life: results from the assessment of spondyloarthritis international society comorbidities in spondyloarthritis study. Arthritis Care Res (Hoboken) 2018; 70: 1257-1262.

9.Charlson M, Szatrowski TP, Peterson J, et al. Validation of a combined comorbidity index. J Clin Epidemiol 1994; 47: 1245-1251.

10. England BR, Sayles H, Mikuls TR, et al. Validation of the Rheumatic Disease Comorbidity Index. Arthritis Care Res 2015; 67: 6865-6872.

11. Radner H, Yoshida K, Mjaavatten MD, et al. Development of a Multimorbidity Index: Impact on quality of life using a rheumatoid arthritis cohort. Semin Arthritis Rheum 2015; 45: 167-173.

12. Pincus T, Askanase AD, Swearingen CJ. A multi-dimensional health assessment questionnaire (MDHAQ) and routine assessment of patient index data (RAPID3) scores are informative in patients with all rheumatic diseases. Rheum Dis Clin North Am 2009; 35: 819-827.

13. Radner H, Yoshida K, Smolen JS, et al. Multimorbidity and rheumatic conditions-enhancing the concept of comorbidity. Nat Rev Rheumatol 2014; 10: 252-256.

14. Baillet A, Gossee L, Carmona L, et al. Points to consider for reporting, screening for and preventing selected comorbidities in chronic inflammatory rheumatic diseases in daily practice: a EULAR initiative. Ann Rheum Dis 2016; 75: 965-973. 